This item was submitted to Loughborough's Research Repository by the author.

Items in Figshare are protected by copyright, with all rights reserved, unless otherwise indicated.

\title{
Enhanced Li-ion dynamics in trivalently doped Lithium Phosphidosilicate Li2SiP2: A candidate material as a solid li electrolyte
}

PLEASE CITE THE PUBLISHED VERSION

https://doi.org/10.1039/c8ta10788b

PUBLISHER

(C) Royal Society of Chemistry (RSC)

VERSION

AM (Accepted Manuscript)

\section{PUBLISHER STATEMENT}

This paper was accepted for publication in the journal Journal of Materials Chemistry A and the definitive published version is available at https://doi.org/10.1039/c8ta10788b.

LICENCE

CC BY-NC-ND 4.0

\section{REPOSITORY RECORD}

Yeandel, Stephen, David O. Scanlon, and Pooja Goddard. 2019. "Enhanced Li-ion Dynamics in Trivalently Doped Lithium Phosphidosilicate Li2sip2: A Candidate Material as a Solid Li Electrolyte". Loughborough University. https://hdl.handle.net/2134/36757. 


\section{Journal of \\ Materials Chemistry A}

\section{Accepted Manuscript}

This article can be cited before page numbers have been issued, to do this please use: S. R. Yeandel, D.

O. Scanlon and P. Goddard, J. Mater. Chem. A, 2019, DOI: 10.1039/C8TA10788B.

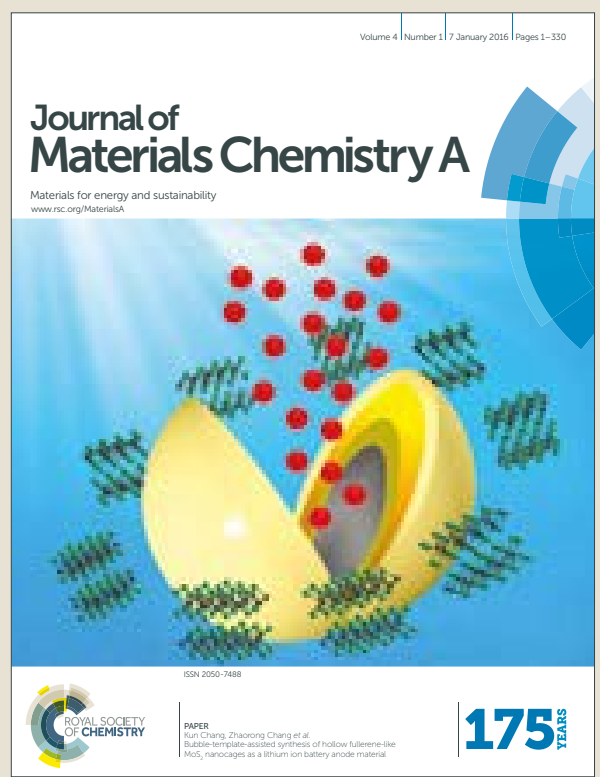

This is an Accepted Manuscript, which has been through the Royal Society of Chemistry peer review process and has been accepted for publication.

Accepted Manuscripts are published online shortly after acceptance, before technical editing, formatting and proof reading. Using this free service, authors can make their results available to the community, in citable form, before we publish the edited article. We will replace this Accepted Manuscript with the edited and formatted Advance Article as soon as it is available.

You can find more information about Accepted Manuscripts in the author guidelines.

Please note that technical editing may introduce minor changes to the text and/or graphics, which may alter content. The journal's standard Terms \& Conditions and the ethical guidelines, outlined in our author and reviewer resource centre, still apply. In no event shall the Royal Society of Chemistry be held responsible for any errors or omissions in this Accepted Manuscript or any consequences arising from the use of any information it contains. 


\title{
Journal Name
}

\section{ARTICLE TYPE}

\section{Cite this: DOI: $10.1039 / x x x x x x x x x x$ Enhanced Li-ion Dynamics in Trivalently Doped
Lithium Phosphidosilicate $\mathrm{Li}_{2} \mathrm{SiP}_{2}:$ A Candidate Mate-
rial as a Solid Li Electrolyte}

\author{
Stephen R. Yeandel, ${ }^{a}$ David O. Scanlon, ${ }^{b c d}$ and Pooja Goddard ${ }^{* a}$
}

Received Date

Accepted Date

DOI: 10.1039/xxxxxxxxxx

www.rsc.org/journalname
Oxide and sulphide solid electrolyte materials have enjoyed significant interest in the solid-state battery community. Phosphide materials however are relatively unexplored despite the potential for being high lithium containing systems. This work reports on the phosphidosilicate system $\mathrm{Li}_{2} \mathrm{SiP}_{2}$, one of many systems in the Li-Si-P phase diagram. The phosphidosilicates display complex structures and very large unit cells, which present challenges for ab-initio simulations. We present the first computational report on the theoretical ionic conductivity and related diffusion mechanisms of the material $\mathrm{Li}_{2} \mathrm{SiP}_{2}$, selected due to it's unusual supertetrahedral framework which is a recurrent motif amongst the phosphidosilicates. Group 13 dopants have also been introduced into $\mathrm{Li}_{2} \mathrm{SiP}_{2}$ showing preference for the silicon site over the lithium site, with $\mathrm{Al}_{S i}^{\prime}$ doping showing extremely low defect incorporation energies of $0.05 \mathrm{eV}$, with no increase in defect energy up to concentrations of $10 \% \mathrm{Al}_{\mathrm{Si}}^{\prime}$. Furthermore, clustering of $\mathrm{Al}_{\mathrm{Si}}^{\prime}$ has been found to be unfavourable, in line with trends seen in oxide zeolite structures. Ab-initio molecular dynamics (AIMD) simulations indicate high ionic conductivity in pure $\mathrm{Li}_{2} \mathrm{SiP}_{2}$ of up to $3.19 \times 10^{-1} \mathrm{~S}_{\mathrm{cm}} \mathrm{cm}^{-1}$ at $700 \mathrm{~K}$. Doping with $10 \% \mathrm{Al}_{\mathrm{Si}}^{\prime}$ and associated $\mathrm{Li}_{\mathrm{i}}^{\bullet}$ compensating defects leads to higher ionic conductivities at lower temperatures when compared to pure $\mathrm{Li}_{2} \mathrm{SiP}_{2}$. The activation energies to lithium diffusion were found to be low at $0.30 \mathrm{eV}$ and $0.24 \mathrm{eV}$ for pure and $10 \% \mathrm{Al}_{\mathrm{Si}}^{\prime}$ doped $\mathrm{Li}_{2} \mathrm{SiP}_{2}$ respectively, in line with previous experimental observations of pure $\mathrm{Li}_{2} \mathrm{SiP}_{2}$. Multiple lithium migration pathways have also been extracted, with some mechanisms displaying activation energies as low as $0.05 \mathrm{eV}$. Furthermore, our calculated intercalation voltages suggest that these materials are stable against lithium metal and therefore could be very attractive in stabilising the electrode/electrolyte interface.

\section{Introduction}

Lithium batteries are a ubiquitous part of modern life and have facilitated a revolution in personal electronics. Safety concerns due to the current liquid electrolyte, however, have inhibited further development of their use as large scale grid storage devices. In order to continue this revolution, new battery materials, architectures and technologies are being developed and the field has gained quite some traction, with all solid state batteries taking

\footnotetext{
${ }^{a}$ Department of Chemistry, Loughborough University, Epinal Way, Loughborough LE11 3TU, UK. E-mail: P.Goddard@lboro.ac.uk

${ }^{b}$ Department of Chemistry, University College London, 20 Gordon Street, London WC1H OAJ, UK.

c Thomas Young Centre, University College London, Gower Street, London WC1E 6BT, UK.

${ }^{d}$ Diamond Light Source Ltd., Diamond House, Harwell Science and Innovation Campus, Didcot, Oxfordshire OX11 ODE, UK.

$\dagger$ Electronic Supplementary Information (ESI) available: [details of any supplementary information available should be included here]. See DOI:
}

centre stage. ${ }^{1-7}$

Solid state batteries have advantages in volumetric energy density relative to current lithium-ion batteries and improved safety due to the chemical stability of solid electrolytes. Li-ion solidstate conductors require high ionic conductivity at room temperature and low activation energy $\left(E_{a}\right)$ for use over a broad range of operating temperatures. In addition, other properties such as electrochemical stability against the anode and cathode, and environmental stability are preferred as they reduce the complexity of the battery. ${ }^{1}$ In the last decade, sulphide systems have been of growing interest due to the discovery of thio-LISICON type lithium superionic conductors with reported ionic conductivity of $12{\mathrm{mS} . \mathrm{cm}^{-1}}^{-}$at room temperature. ${ }^{2-4}$

More recently, a range of lithium phosphidosilicate systems have been reported as promising candidates for solid Li ion conducting electrolytes due to their high Li compositions. ${ }^{8-10}$ The high negative charge of the phosphide anion allows a higher cation $\mathrm{Li}^{+}$content which leads to unique crystal structures which 
may also exhibit high Li ion diffusion. A list of the structure types adopted by the alkali metal phosphidosilicates is reported in Table 1 highlighting the complex relationship between the different crystal structures and activation energies and hence Li ion diffusion.

In 2016 Haffner et al. ${ }^{8}$ reported the synthesis of two new materials, $\mathrm{Li}_{2} \mathrm{SiP}_{2}$ and $\mathrm{LiSi}_{2} \mathrm{P}_{3}$. Both materials contain large interlinked networks of supertetrahedra of silicon and phosphorous $\left(\mathrm{SiP}_{4}\right)$. The charge compensating lithium ions are located within the spaces between the supertetrahedra. The lithium ion diffusion in both of these materials was investigated by temperaturedependent ${ }^{7} \mathrm{Li}$ solid-state MAS NMR spectroscopy and reported to be very competitive with Li migration barriers as low as $0.1 \mathrm{eV}$ for $\mathrm{Li}_{2} \mathrm{SiP}_{2}$ and $0.07 \mathrm{eV}$ for $\mathrm{LiSi}_{2} \mathrm{P}_{3}$, making them promising candidates for solid Li electrolyte applications.

Soon after, Toffoletti et al. ${ }^{9}$ elucidated the structure of another lithium containing phosphidosilicate, $\mathrm{Li}_{8} \mathrm{SiP}_{4}$, along with confirmation of the $\mathrm{Li}_{2} \mathrm{SiP}_{2}$ structure. These systems were found to have ionic conductivities of between $1.15 \times 10^{-6} \mathrm{Scm}^{-1}$ at $0{ }^{\circ} \mathrm{C}$ and $1.2 \times 10^{-4} \mathrm{Scm}^{-1}$ at $75{ }^{\circ} \mathrm{C}$ for $\mathrm{Li}_{8} \mathrm{SiP}_{4}$ and between $6.1 \times 10^{-8} \mathrm{Scm}^{-1}$ at $0{ }^{\circ} \mathrm{C}$ and $6.0 \times 10^{-6} \mathrm{Scm}^{-1}$ at $75{ }^{\circ} \mathrm{C}$ for $\mathrm{Li}_{2} \mathrm{SiP}_{2}$. The energy barriers to lithium diffusion were also determined by temperature-dependent ${ }^{7} \mathrm{Li}$ solid-state MAS NMR spectroscopy and found to be $0.373 \mathrm{eV}$ for $\mathrm{Li}_{8} \mathrm{SiP}_{4}$ and $0.487 \mathrm{eV}$ for $\mathrm{Li}_{2} \mathrm{SiP}_{2} \cdot{ }^{9}$ However, Haffner et al. report an activation energy of $0.1 \mathrm{eV}$ for $\mathrm{Li}_{2} \mathrm{SiP}_{2}$, indicating the sensitivity of this system to synthesis conditions.

Two more materials in the Li-Si-P system have also recently been reported by the same group, $\mathrm{Li}_{10} \mathrm{Si}_{2} \mathrm{P}_{6}$ and $\mathrm{Li}_{3} \mathrm{Si}_{3} \mathrm{P}_{7} \cdot{ }^{10}$ The interesting chemistry within the Li-Si-P systems is extremely varied with several structures showing high lithium content and low energy barriers to lithium diffusion and therefore ideal for a computational study such as this work. In this work we focus on $\mathrm{Li}_{2} \mathrm{SiP}_{2}$ (LSP) where the structure is well known and manageable in terms of crystal structure size and computational expense.

\subsection{Structure of $\mathrm{Li}_{2} \mathrm{SiP}_{2}$ (LSP)}

In LSP the framework is comprised of corner-linked $\mathrm{Si}_{4} \mathrm{P}_{10}{ }^{14-}$ supertetrahedra, which are then assembled into two entwined 3D networks, see Figure 1. Each of these 3D networks has the same structure as the $\mathrm{SiP}_{4}{ }^{8-}$ tetrahedra formed in the chalcopyrite structured material $\mathrm{MgSiP}_{2}{ }^{15}$, but with $\mathrm{Si}_{4} \mathrm{P}_{10}{ }^{14-}$ replacing $\mathrm{SiP}_{4}{ }^{8-}$. The charge balancing $\mathrm{Li}^{+}$occupy the interstitial spaces of the entwined networks in clearly defined locations, however unoccupied interstitial sites are also available (based on the $\mathrm{Li}_{2} \mathrm{SiN}_{2}$ structure). ${ }^{8,16}$ Experimentally the energy barriers to lithium diffusion in LSP were measured to be between 0.1-0.487 eV, however some disagreement remains about the exact value of the energy barrier, Table $1.8,9$

There is one symmetrical $\mathrm{Si}^{4+}$ site (32g), situated within the supertetrahedra of the LSP structure, Figure 2 a). In contrast, three distinct $\mathrm{Li}^{+}$sites are identified in the interstitial space. $\mathrm{Li}^{+}$site 1 $(16 f)$ is located at the centre of a $\mathrm{LiP}_{4}{ }^{11-}$ tetrahedra, with the $\mathrm{P}^{3-}$ ions being located at the edges of different $\mathrm{Si}_{4} \mathrm{P}_{10}{ }^{14-}$ supertetrahedra within the structure. $\mathrm{Li}^{+}$site $2(32 g)$ is located at the centre

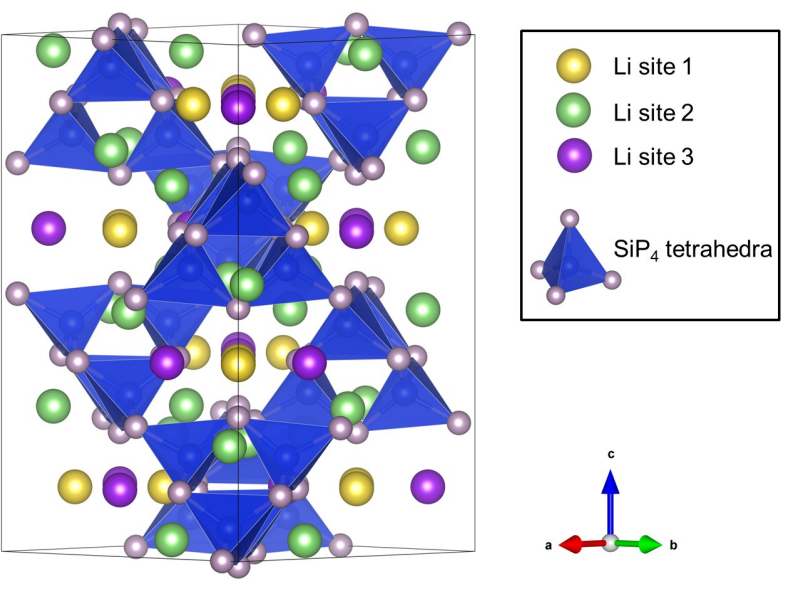

Fig. 1 The structure of $\mathrm{Li}_{2} \mathrm{SiP}_{2}$ showing the intertwined 3D networks with Li distributed in the interstitial spaces.

of a distorted $\mathrm{LiP}_{4}{ }^{11-}$ tetrahedra, with three $\mathrm{P}_{3}{ }^{-}$ions comprising the face of a supertetrahedra. $\mathrm{Li}^{+}$site $3(16 f)$ shares a channel with the $\mathrm{Li}^{+}$site 1 and is also 4-fold coordinated but in a buckled square-planar arrangement.

\section{Methodology}

\subsection{DFT methodology}

All our DFT calculations were performed using the Vienna Ab initio Simulation Package (VASP) ${ }^{17}$ using the projector augmented wave (PAW) method ${ }^{18}$ and the PBEsol ${ }^{19}$ functional. All calculations were spin polarised. Long-range dispersion corrections have not been included as the constituent ions are relatively small and such interactions will be effectively screened in a condensed solid. Lattice vectors were allowed to vary during minimisations and a force tolerance of $1.0 \times 10^{-2} \mathrm{eV} . \AA^{-1}$ was applied for convergence. The calculated lattice parameters are all within $1 \%$ of the experimental lattice parameters determined by both Toffoletti et al. ${ }^{9}$ and Haffner et al. ${ }^{8}$. Further details of the computational parameters are available in the SI.

Final lattice energies were obtained by single point calculation on the optimised structures, using the more accurate reciprocal space projection scheme ${ }^{17}$ with electronic convergence set to a tolerance of $1.0 \times 10^{-8} \mathrm{eV}$. As the calculation of defect energies requires taking the difference of two very large numbers, the computed lattice energies must be extremely precise. To this end, we have selected a very high plane wave energy cutoff of $1000 \mathrm{eV}$ for all calculations unless stated otherwise.

To ensure calculated defect energies are accurate and not influenced by finite size effects, three sizes of LSP supercell were created. These supercells contain 160, 320 and 640 atoms and are referred to as LSP-160, LSP-320 and LSP-640 respectively see SI). All materials and supercells had their convergence with respect to plane wave energy cutoff and k-point grid tested, optimised settings are available in the SI. In order to simplify the defect calculations, compensating defects were introduced into the same simulation cell as nearest neighbours to the dopant species. This reduces the configurations which require checking, but means that the defect energies quoted include a contribution due to defect 
Table 1 Framework structures, space groups and activation energies of various Alkali metal phosphidosilicates reported in the literature.

\begin{tabular}{|c|c|c|c|c|}
\hline Material & Framework structure & Space group & Activation energy $(\mathrm{eV})$ & Reference \\
\hline$\overline{\mathrm{LiSi}_{2} \mathrm{P}_{3}}$ & Supertetrahedral framework & $I 4_{1} / a(88)$ & 0.07 & 8 \\
\hline $\mathrm{Li}_{2} \mathrm{SiP}_{2}$ & Supertetrahedral framework & $I 4_{1} /$ acd $(142)$ & $0.10-0.487$ & 8,9 \\
\hline $\mathrm{Li}_{8} \mathrm{SiP}_{4}$ & Isolated $\mathrm{SiP}_{4}{ }^{8-}$ tetrahedra & $P a \overline{3}(205)$ & 0.373 & 9 \\
\hline $\mathrm{Li}_{3} \mathrm{Si}_{3} \mathrm{P}_{7}$ & Corner linked $\mathrm{SiP}_{4}{ }^{8-}$ tetrahedra with P-P bonds & $P 2_{1} / m(11)$ & not reported & 10 \\
\hline $\mathrm{Li}_{10} \mathrm{Si}_{2} \mathrm{P}_{6}$ & Isolated $\mathrm{Si}_{2} \mathrm{P}_{6}{ }^{10-}$ double tetrahedra & $P 2_{1} / n(14)$ & not reported & 10 \\
\hline $\mathrm{Na}_{10} \mathrm{Si}_{2} \mathrm{P}_{6}$ & Isolated $\mathrm{Si}_{2} \mathrm{P}_{6}{ }^{10-}$ double tetrahedra & $I 4_{1} /$ acd (14) & not reported & 11 \\
\hline $\mathrm{Na}_{19} \mathrm{Si}_{13} \mathrm{P}_{25}$ & Supertetrahedral framework & $P \overline{1}(2)$ & 0.36 & 12 \\
\hline $\mathrm{Na}_{23} \mathrm{Si}_{19} \mathrm{P}_{33}$ & Supertetrahedral framework & $C 2 / c(15)$ & 0.47 & 12 \\
\hline $\mathrm{Na}_{23} \mathrm{Si}_{28} \mathrm{P}_{45}$ & Supertetrahedral framework & $P 2_{1} / c(14)$ & 0.38 & 12 \\
\hline $\mathrm{Na}_{23} \mathrm{Si}_{37} \mathrm{P}_{57}$ & Supertetrahedral framework & $C 2 / c(15)$ & 0.37 & 12 \\
\hline$L T-\mathrm{NaSi}_{2} \mathrm{P}_{3}$ & Supertetrahedral framework & $I 4_{1} / a(88)$ & 0.23 & 12 \\
\hline$H T-\mathrm{NaSi}_{2} \mathrm{P}_{3}$ & Supertetrahedral framework & $I 4_{1} /$ acd $(142)$ & 0.25 & 12 \\
\hline $\mathrm{K}_{2} \mathrm{SiP}_{2}$ & $1 \mathrm{D} \mathrm{SiP}{ }_{2}^{2-}$ chains & $\operatorname{Ibam}(72)$ & not reported & 13 \\
\hline $\mathrm{KSi}_{2} \mathrm{P}_{3}$ & $2 \mathrm{D} \mathrm{Si}_{2} \mathrm{P}_{3}{ }^{1-}$ layers & $C 2 / c(15)$ & not reported & 14 \\
\hline
\end{tabular}

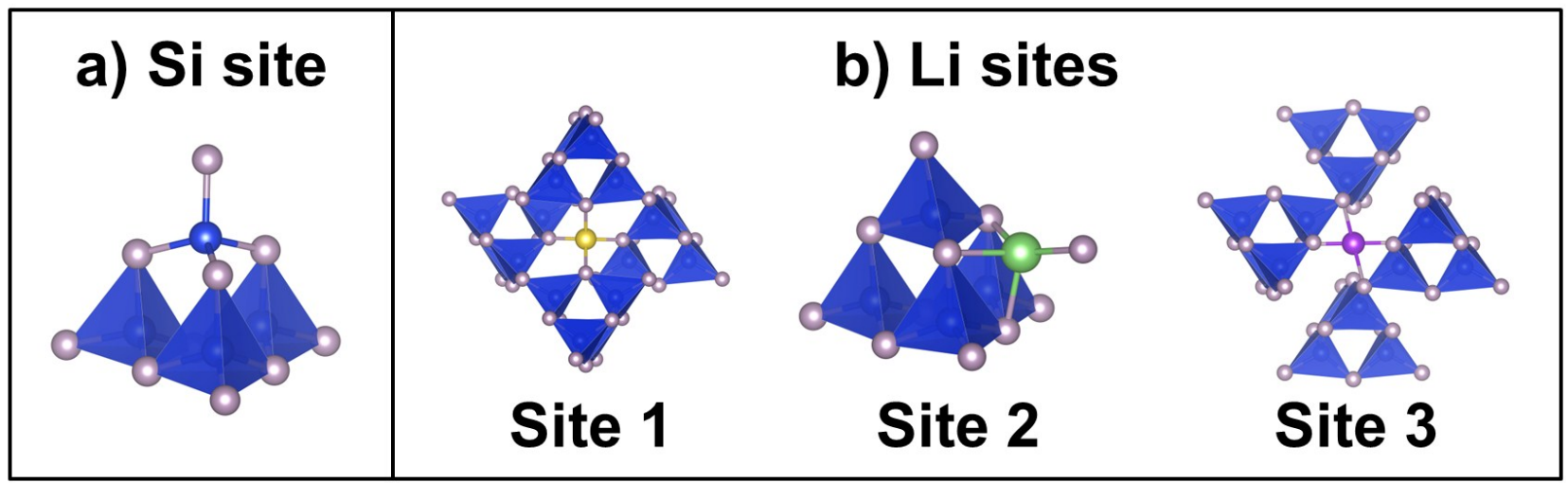

Fig. 2 Two cation sites available within $\mathrm{Li}_{2} \mathrm{SiP}_{2}$, a) a single $\mathrm{Si}$ site and b) three distinct $\mathrm{Li}$ sites. For legend see Figure 1.

clustering.

To obtain more accurate electronic properties, such as the band gap, electronic Density of States (DOS) and intercalation/deintercalation voltages a hybrid functional, HSE06, ${ }^{20}$ was used instead of the PBEsol GGA functional. The DOS were calculated using a single point HSE06 calculation at the full $3 \times 3 \times 2$ k-point grid with a $1000 \mathrm{eV}$ plane wave cutoff. The lithium intercalation/deintercalation voltages are also calculated using the HSE06 functional, but use only the $\Gamma$-point and a reduced $700 \mathrm{eV}$ cutoff. All hybrid HSE06 calculations were performed on the LSP160 atom simulation cell.

\subsection{Ab-initio Molecular dynamics (AIMD)}

Since energy minimisation methods yield the lattice constants of the LSP systems equivalent to those of a $0 \mathrm{~K}$ system, neglecting zero-point motion, a different approach must be used to obtain the lattice constants at temperature. The correct volume at each temperature was obtained using Langevin dynamics ${ }^{21}$ via AIMD. The Parrinello and Rahman was employed in this work. ${ }^{22,23}$ In this approach, the atomic velocities are adjusted via a stochastic process emulating Brownian motion of a fictional solvent, Equation 1.

$$
\dot{p}_{i}=F_{i}-\gamma_{i} p_{i}+f_{i}
$$

the momentum, $\dot{p}_{i}$ is the time derivative of the momentum, $f_{i}$ is a random force. The random force, $f_{i}$, has dispersion $\sigma_{i}$ and is related to the friction coefficient by Equation 2 .

$$
\sigma_{i}^{2}=\frac{2 m_{i} \gamma k_{B} T}{\Delta t}
$$

By tuning the coupling parameters of the Langevin dynamics, the process acts as a thermostat and is able to control the temperature of the real system, thereby approximating a canonical ensemble. Additionally, the lattice degrees of freedom are also coupled to the Langevin equations, allowing them to evolve over time. The advantage of this approach is that the fluctuations of the lattice degrees of freedom may be carefully controlled, counterbalancing the large fluctuations expected when a system of less than 1,000 atoms is considered.

Although Langevin dynamics are useful for obtaining the correct instantaneous averages, it fails to obtain correct timecorrelated quantities such as diffusion coefficients. This stems from the random stochastic variable used to control the temperature. To obtain the correct diffusion coefficients for these systems a Nosé-Hoover thermostat is employed for the next stage of the canonical simulation. ${ }^{24,25}$ From this data it is possible to extract a Mean Squared Displacement (MSD) for each species, using Equation 3.

$$
\operatorname{MSD}(t)=\left\langle\left(\vec{r}_{i}(t)-\vec{r}_{i}(0)\right)^{2}\right\rangle
$$


where $\vec{r}_{i}(t)$ is the position vector at time $t$ and likewise $\vec{r}_{i}(0)$ is the initial position. Angled brackets denote an average employing a shifting time origin which can be used to further improve accuracy. The diffusion coefficients can then be extracted via the Einstein diffusion equation, Equation 4.

$$
\operatorname{MSD}(t)=6 D t+C
$$

where $D$ is the diffusion coefficient and $C$ is a constant. All AIMD simulations were performed in the LSP-320 simulation cell. A timestep of $0.5 \mathrm{fs}$ was used for all AIMD simulations, with a reduced k-point grid utilising only the $\Gamma$-point and a reduced electronic cutoff of $700 \mathrm{eV}$.

\section{Results}

\subsection{Intrinsic defects}

The most important intrinsic defect to consider in pure LSP with regards to lithium migration is the lithium Frenkel defect.

The Li Frenkel defect energy was calculated by taking an observed spontaneous Frenkel from the AIMD data and forming the same defect in a pure LSP-160 simulation cell. The pathway chosen was that of a site 1 lithium moving into an interstitial space in the site 2 pathway, and a site 3 lithium immediately migrating onto the vacant site 1 . This observed Frenkel formation gives a defect energy of $0.769 \mathrm{eV}$. When the separation of the vacancyinterstitial pair is maximised within the supercell, retaining the same vacancy and interstitial sites, the defect energy increases to approximately $0.92 \mathrm{eV}$. The lithium Frenkel defect energies are relatively high compared to the $\mathrm{Al}_{\mathrm{Si}}^{\prime}$ and $\mathrm{Li}_{\mathrm{i}}^{\bullet}$ defect pair energy calculated from Equation 5, indicating it is the lithium vacancy which is the greatest contribution to the lithium Frenkel energy.

\subsection{Trivalent doping}

To date, there has been little work done on the defect chemistry of the phosphidosilicates, however inspiration may be taken from zeolites which also demonstrate complex frameworks, although are more porous. The zeolites frequently incorporate aluminium defects into their structures ${ }^{26,27}$, although boron and gallium are also known to incorporate in some cases ${ }^{28-30}$. We have therefore opted to study the doping of $\mathrm{Li}_{2} \mathrm{SiP}_{2}$ with group 13 elements. In particular group 13 phosphides have been selected as the source of dopant ions as this greatly simplifies the possible defect compensating mechanisms. Thus, $\mathrm{BP}^{31}, \mathrm{AlP}^{32}$ and $\mathrm{GaP}^{33}$, all Zinc blende structures, were selected as the source of dopant ions.

Doping of $\mathrm{Li}_{2} \mathrm{SiP}_{2}$ may occur on the $\mathrm{Si}^{4+}$ site or the three distinct $\mathrm{Li}^{+}$sites. Two compensating mechanisms have been considered and are given in Equations 5 and 6.

$$
\begin{gathered}
\mathrm{MP}+\mathrm{Li}_{3} \mathrm{P}+\mathrm{Si}_{\mathrm{Si}}^{\mathrm{x}} \longrightarrow \mathrm{M}_{\mathrm{Si}}^{\prime}+\mathrm{Li}_{\mathrm{i}}^{\bullet}+\mathrm{Li}_{2} \mathrm{SiP}_{2} \\
\mathrm{MP}+3 \mathrm{Li}_{\mathrm{Li}}^{\mathrm{x}} \longrightarrow \mathrm{M}_{\mathrm{Li}}^{\bullet \bullet}+2 \mathrm{~V}_{\mathrm{Li}}^{\prime}+\mathrm{Li}_{3} \mathrm{P}
\end{gathered}
$$

Equation 5 represents a group 13 element (M) doping on a silicon site, Figure 2 a), with the formation of a compensating $\mathrm{Li}_{\mathrm{i}}^{\bullet}$ defect. As all $\mathrm{Si}^{4+}$ sites are equivalent under symmetry, the possible configurations are limited. Doping using Equation 6 is more challenging as there are three distinct $\mathrm{Li}^{+}$sites, each of which must be considered individually, Figure $2 \mathrm{~b}$ ).

The defective LSP systems were created by taking the supercells outlined in the SI and inserting a single dopant atom on either the $\mathrm{Si}^{4+}$ site or the $\mathrm{Li}^{+}$site.

The calculated defect energies for doping LSP with $\mathrm{B}^{3+}, \mathrm{Al}^{3+}$ and $\mathrm{Ga}^{3+}$ using Equations 5 and 6 are presented in Table 2

Table 2 Defect energies using Equations 5 and 6

\begin{tabular}{lllll}
\hline Site & Supercell & $\mathrm{B}^{3+}(\mathrm{eV})$ & $\mathrm{Al}^{3+}(\mathrm{eV})$ & $\mathrm{Ga}^{3+}(\mathrm{eV})$ \\
\hline \multirow{2}{*}{ Si site } & LSP-160 & 1.208 & 0.044 & 0.173 \\
& LSP-320 & 1.226 & 0.049 & 0.180 \\
& LSP-640 & 1.225 & 0.051 & 0.182 \\
\hline \multirow{2}{*}{ Li site 1} & LSP-160 & 4.307 & 1.947 & 2.076 \\
& LSP-320 & 4.362 & 1.974 & 2.100 \\
& LSP-640 & 4.352 & 1.961 & 2.087 \\
\hline \multirow{2}{*}{ Li site 2 } & LSP-160 & 3.682 & 1.976 & 2.084 \\
& LSP-320 & 3.714 & 1.969 & 2.077 \\
& LSP-640 & 3.744 & 1.970 & 2.076 \\
\multirow{2}{*}{ Li site 3} & LSP-160 & 3.598 & 2.512 & 2.579 \\
& LSP-320 & 3.674 & 2.528 & 2.584 \\
& LSP-640 & 3.679 & 2.508 & 2.566
\end{tabular}

The calculated defect energies indicate that trivalent doping is clearly more favourable on the $\mathrm{Si}^{4+}$ site than the $\mathrm{Li}^{+}$site. Within the $\mathrm{Si}^{4+}$ site, $\mathrm{Al}^{3+}$ displays extremely low defect energies of $\sim 0.05 \mathrm{eV}$ which suggests there may be significant incorporation of aluminium in this material if a suitable source is available during synthesis. $\mathrm{Ga}^{3+}$ doping on $\mathrm{Si}^{4+}$ is the next most favourable doping strategy with a defect energy of $\sim 0.18 \mathrm{eV}$, while doping with $\mathrm{B}^{3+}$ on $\mathrm{Si}^{4+}$ is the least favourable strategy using Equation 5 with a defect energy of $\sim 1.22 \mathrm{eV}$.

The low incorporation energy of aluminium into the LSP structure, with reduced favourability of boron and gallium incorporation, mirrors the trends observed in zeolites. Aluminium frequently substitutes into zeolite structures of all types. ${ }^{26,27,34} \mathrm{Al}-$ though gallium also frequently incorporates to a significant degree, the larger ionic radius results in longer bond lengths and the preference to form slightly different zeolite structures. ${ }^{30,34}$ This is mirrored in our results which show gallium is a less favourable dopant of LSP than aluminium, but still has a low enough defect energy to be able to incorporate, Table 2. Furthermore, boron has a significantly smaller ionic radius which leads to significantly shorter bond lengths and poor substitution for aluminium in zeolite frameworks. ${ }^{28,34}$ Once again this is reflected in our results which show the defect energy of incorporating boron into the LSP structure is significantly higher than either aluminium or gallium, Table 2.

Doping on the $\mathrm{Li}^{+}$sites shows more variability between the dopant species, Table 2. Once again $\mathrm{Al}^{3+}$ is the most favourable dopant across all $\mathrm{Li}^{+}$sites, with the tetrahedral sites 1 and 2 preferred $(\sim 1.97 \mathrm{eV})$ over the non-tetrahedral site $3(\sim 2.51 \mathrm{eV})$. $\mathrm{Ga}^{3+}$ doping follows much the same trend as $\mathrm{Al}^{3+}$ doping with defect energies of $\sim 2.07 \mathrm{eV}$ across tetrahedral sites 1 and 2 while the non-tetrahedral site 3 has a defect energy of $\sim 2.57 \mathrm{eV}$. Interestingly, $\mathrm{B}^{3+}$ doping on the $\mathrm{Li}^{+}$sites does not follow this trend and instead the non-tetrahedral site 3 is the most stable $\mathrm{Li}^{+}$site defect $(\sim 3.67 \mathrm{eV})$ with site 2 being of a similar energy $(\sim 3.73 \mathrm{eV})$ 
and site 1 being considerably higher $(\sim 4.36 \mathrm{eV})$. This unusual reversal of the favoured doping sites is likely due to the small size of the $\mathrm{B}^{3+}$ cation $^{35}$ and larger distortion of the four supertetrahedra coordinating $\mathrm{Li}^{+}$site 1 , while sites 2 and 3 involve a smaller distortion of the framework in order to fully coordinate the $\mathrm{B}^{3+}$ dopant.

\subsection{Defect clustering}

To probe the limit of $\mathrm{Al}_{\mathrm{Si}}^{\prime}$ dopant clustering, nine different systems were created using the LSP-320 simulation cell, each with six randomly distributed $\mathrm{Al}_{\mathrm{Si}}^{\prime}$ defects. An additional system was created with four $\mathrm{Al}_{\mathrm{Si}}^{\prime}$ defects forming a whole supertetrahedra, with the remaining two $\mathrm{Al}_{\mathrm{Si}}^{\prime}$ defects placed randomly in the system. The resulting concentration of $\mathrm{Al}_{\mathrm{Si}}^{\prime}$ defects in these systems is $9.375 \%$. Charge compensating $\mathrm{Li}^{+}$interstitial defects have not been included in these calculations as this would introduce a secondary configurational contribution to the energy which would be extremely challenging to disentangle from the effects of aluminium clustering.. However, the total number of electrons in these calculations has been set so that the supertetrahedral frameworks retain the correct charge. This setup ensures the limit of $\mathrm{Al}_{\mathrm{Si}}^{\prime}$ clustering is thoroughly probed and that the energy differences between the systems are due to the effects of clustering of $\mathrm{Al}_{\mathrm{Si}}^{\prime}$ defects alone, excluding the effects of compensating $\mathrm{Li}_{\mathrm{i}}^{\circ}$ species. An example simulation cell is given in Figure 3.

To measure the degree of $\mathrm{Al}_{\mathrm{Si}}^{\prime}$ clustering in $\mathrm{Al}$ doped LSP, we consider only adjacent $\mathrm{Si}^{4+}$ sites, the degree of clustering may be given by the number of $\mathrm{P}^{3-}$ ions shared by $\mathrm{Al}^{3+}$ ions. Plotting the relative lattice energies against this metric gives the graph in Figure 4.

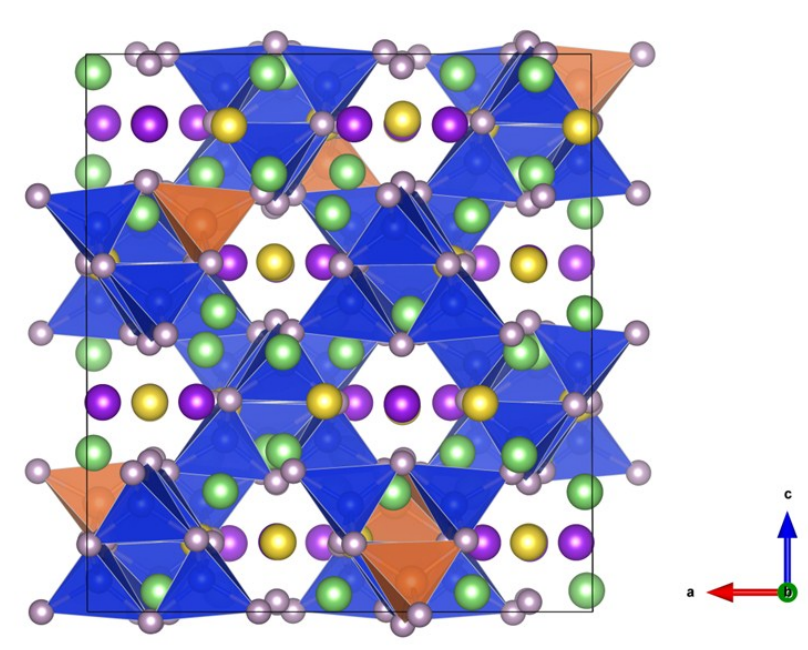

Fig. 3 Example LSP-320 simulation cell with $6 \mathrm{Al}_{\mathrm{Si}}^{\prime}$ defects (orange tetrahedra).

These results show a clear trend of higher clustering of $\mathrm{Al}_{\mathrm{Si}}^{\prime}$ resulting in less stable systems. However, the energy per $\mathrm{Al}_{\mathrm{Si}}^{\prime}$ defect is small. The most stable system has the $\mathrm{Al}_{\mathrm{Si}}^{\prime}$ defects distributed evenly in space and across the two supertetrahedral frameworks. In contrast, the additional system constructed as to have a single superterahedra containing only $\mathrm{Al}_{\mathrm{Si}}^{\prime}$ defects is the least stable.

By inserting the missing $\mathrm{Li}_{\mathrm{i}}^{\circ}$ interstitials into the most stable $\mathrm{Al}_{\mathrm{Si}}^{\prime}$ doped system, the defect energy can be calculated as before. Ordering of $\mathrm{Li}_{i}^{\bullet}$ interstitials is now less certain due to the random placement of $\mathrm{Al}_{\mathrm{Si}}^{\prime}$ defects and so the $\mathrm{Li}_{\mathrm{i}}^{\bullet}$ interstitials are inserted randomly.

The defect energies from these calculations are thus not exactly comparable to those presented in Table 2 but may act as an upper bound on the defect energy in these highly doped systems. Performing this calculation gives a defect energy of $0.062 \mathrm{eV}$ per $\mathrm{Al}_{\mathrm{Si}}^{\prime}$ and $\mathrm{Li}_{\mathrm{i}}^{\bullet}$ pair. This energy is only $0.01 \mathrm{eV}$ higher than for the case of a single $\mathrm{Al}_{\mathrm{Si}}^{\prime}$ defect in an equivalently sized cell (Table 2), indicating tolerance to high defect concentrations in $\mathrm{Li}_{2} \mathrm{SiP}_{2}$.

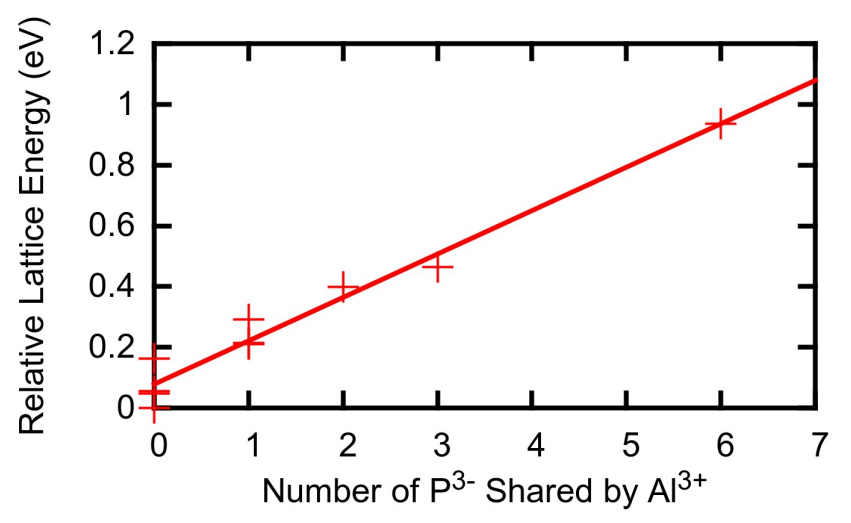

Fig. 4 Relative lattice energies of $\mathrm{Al}_{\mathrm{Si}}^{\prime}$ doped systems against number of shared $\mathrm{P}^{3-}$ ions.

\subsection{Lithium diffusion}

To investigate the lithium diffusion properties within the pure and Al-doped LSP systems, ab-initio molecular dynamics (AIMD) calculations were performed. Four systems in total were simulated; two pure $\mathrm{Li}_{2} \mathrm{SiP}_{2}$ and two $\mathrm{Al}_{\mathrm{Si}}^{\prime}$ doped systems. The Al doped systems selected were the globally most stable and the most stable with one shared $\mathrm{P}^{3-}$ ion. Different starting velocities were used for each systems during initialisation to ensure different regions of phase space were explored.

An AIMD calculation using a Langevin thermostat was performed on each of the 4 systems at 5 different temperatures, for a total duration of 5 ps each. The average lattice vectors were calculated by taking the second half of the Langevin AIMD simulations and averaging the lattice vectors over time. The average cell volume produced for the pure and doped systems via Langevin MD are presented in the SI.

Using the results of the Langevin dynamics the average volume of each system at equilibrium was obtained. The volumes of the two pure systems can be considered to be probing different regions of the same phase space and so have been averaged together. However, The equilibrium volumes for the two doped systems are for different defect configurations and so are probing different phase spaces, and are thus kept separate.

The volume scaled lattice parameters were next applied to the 
simulation cells and kept fixed for the next phase of calculation. A Nosé-Hoover thermostat was also employed instead of a Langevin thermostat and the simulations were run for a further 5 ps each. The velocities were then discarded to remove spurious contributions introduced by the rescaling of the lattice vectors. An additional 20 ps of AIMD were then performed. The calculated MSDs indicate significant movement of lithium ions, but no movement of either silicon or phosphide ions, indicating the system is stable even at $700 \mathrm{~K}$. The full MSDs are available in the SI. The Li component of the MSDs, averaged across the pure or defective systems, are shown in Figure 5.

a)

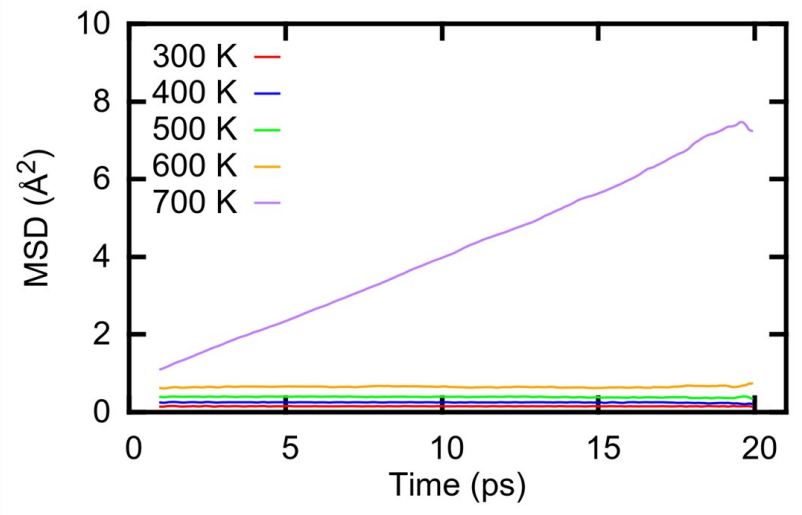

b)

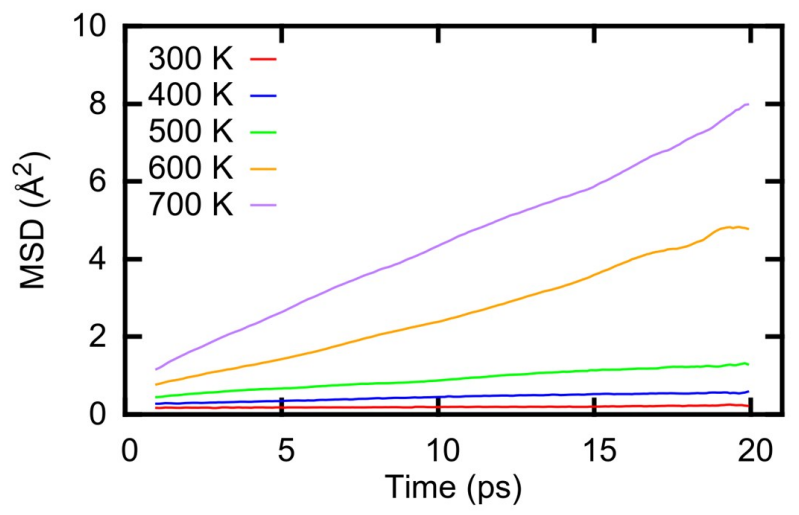

Fig. $5 \mathrm{MSD}$ of lithium at all temperatures for a) pure and b) $\mathrm{Al}_{\mathrm{Si}}^{\prime}$ doped LSP.

Pure LSP, Figure 5 a), shows significant Li diffusion only at $700 \mathrm{~K}$. However, the diffusion of $\mathrm{Li}$ in $\mathrm{Al}_{\mathrm{Si}}^{\prime}$ doped LSP, Figure $5 \mathrm{~b}$ ), is significantly increased down to $500 \mathrm{~K}$. The increase in Li diffusion may be attributed to the inclusion of $\mathrm{Li}_{\mathrm{i}}^{\bullet}$ defects.

The ionic conductivity measured experimentally includes correlated motion of the diffusing species which is absent when the tracer diffusion coefficient is measured via the MSD. The experimentally measured ionic conductivities may be related to the computed diffusion coefficients via a simple ratio, often referred to as a Haven ratio. ${ }^{36}$ The relationship between computed tracer diffusion coefficients and the ionic conductivity is given by Equation 7.

$$
\sigma=\frac{D C q^{2}}{k_{B} T} \frac{1}{H_{R}}
$$

where $D$ is the tracer diffusion coefficient obtained from AIMD via the MSDs, $C$ is the concentration of the diffusing species (lithium ions), $q$ is the charge of the diffusing species, $H_{R}$ is the Haven ratio, $T$ is the temperature and $k_{B}$ is the Boltzmann constant. 37,38

We have adopted a Haven ratio of $1.0 \mathrm{for}^{\mathrm{Al}_{\mathrm{Si}}}$ doped LSP based on the dominance of Li interstitial defects ${ }^{39}$ and therefore suppression of vacancy formation and diffusion. For simplicity we have also adopted a value of 1.0 for the pure LSP system. Both the diffusion coefficients and ionic conductivities are given in Table 3 .

The ionic conductivity of LSP was measured experimentally by Toffoletti et al. ${ }^{9}$ within the range $273.15-348.15 \mathrm{~K}$. In order to compare our computed ionic conductivities we have extrapolated the experimental values into the range $300-700 \mathrm{~K}$. The extrapolation produces an overall excellent agreement between experimental and computed ionic conductivity of pure LSP, with the sole exception of the computed value at $300 \mathrm{~K}$ which is much higher than expected. It must be noted, however, that calculation of diffusion at lower temperatures is challenging using AIMD due to the relatively few diffusion events which are occurring within the simulated timeframe, leading to large uncertainties in the diffusion coefficients. Indeed, examination of Figure 6 shows that pure LSP at $300 \mathrm{~K}$ is the only value significantly deviating from a linear trend.

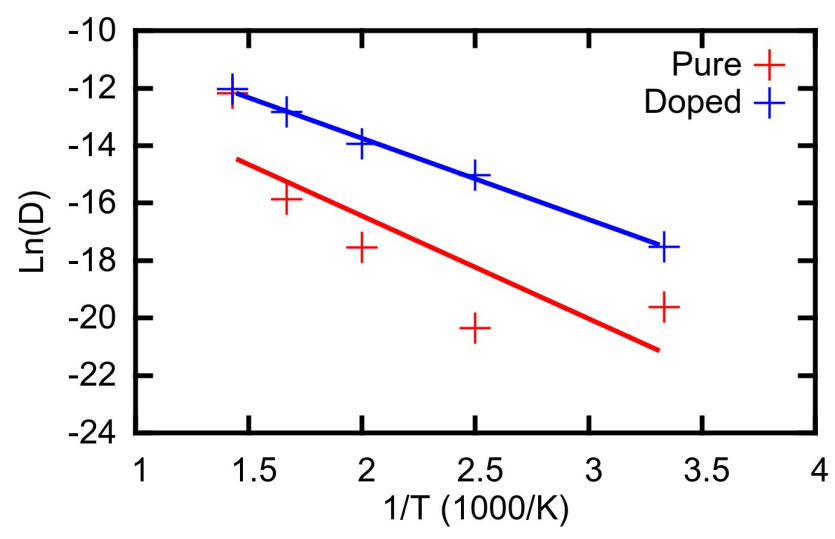

Fig. 6 Arrhenius plot for pure and $\mathrm{Al}_{\mathrm{Si}}^{\prime}$ doped LSP.

The Arrhenius plot also displays good linearity for the $\mathrm{Al}_{\mathrm{Si}}^{\prime}$ doped system. At temperatures below $700 \mathrm{~K}$ the $\mathrm{Al}_{\mathrm{Si}}^{\prime}$ doped systems display 1-2 orders of magnitude higher diffusion. The activation energies extracted from Figure 6 were $0.30 \mathrm{eV}$ for pure $\mathrm{Li}_{2} \mathrm{SiP}_{2}$ and $0.24 \mathrm{eV}$ for $\mathrm{Al}_{\mathrm{Si}}^{\prime}$ doped $\mathrm{Li}_{2} \mathrm{SiP}_{2}$. It is interesting to note that taking only the highly linear portion of the Arrhenius plot between $400-600 \mathrm{~K}$ for pure LSP results in an activation energy of $0.47 \mathrm{eV}$, almost identical to the experimental value of $0.487 \mathrm{eV} .{ }^{9}$

The high diffusion coefficients observed in pure LSP at $700 \mathrm{~K}$ is rather unusual and cannot be simply explained as poor statistical averaging. By inspecting the trajectory of a pure $700 \mathrm{~K}$ AIMD simulation at least one high throughput lithium conduction pathway can be identified, Figure 7. Utilisation of this pathway during the 
Table 3 Calculated diffusion coefficients and ionic conductivity of pure and Al doped LSP

\begin{tabular}{llll}
\hline & Diffusion coefficient $\left(\mathrm{cm}^{2} . \mathrm{s}^{-1}\right)$ & & \multicolumn{2}{l}{$\begin{array}{l}\text { Ionic conductivity }\left(\mathrm{S} . \mathrm{cm}^{-1}\right) \\
\text { Pure LSP }\end{array}$} \\
\hline Temperature (K) & Pure LSP & $2.44 \times 10^{-8}$ & $4.36 \times 10^{-4}$ \\
400 & $3.01 \times 10^{-9}$ & $3.00 \times 10^{-7}$ & $1.58 \times 10^{-4}$ \\
500 & $1.45 \times 10^{-9}$ & $8.74 \times 10^{-7}$ & $2.07 \times 10^{-3}$ \\
500 & $2.38 \times 10^{-8}$ & $2.67 \times 10^{-6}$ & $9.38 \times 10^{-3}$ \\
600 & $1.29 \times 10^{-7}$ & $5.98 \times 10^{-6}$ & $3.19 \times 10^{-1}$ \\
700 & $5.14 \times 10^{-6}$ & & $3.96 \times 10^{-3}$ \\
\end{tabular}

simulation may explain the sudden increase in diffusion at $700 \mathrm{~K}$. This pathway may not be active all the time, which would lead to a reduced diffusion coefficient if pure LSP is simulated over longer timescales.

The pathway shown in Figure 7 b) corresponds to a highly curved 1-dimensional chain of $\mathrm{Li}^{+}$site 2, Figure 7 a). These pathways occur in parallel with a given layer of the $\mathrm{Li}_{2} \mathrm{SiP}_{2}$ structure, and then reoccur rotated by 90 degrees in the layers above and below. Utilisation of this pathway by lithium ions was observed multiple times in several of the AIMD simulations, indicating it may be the origin of the high conductivity observed in $\mathrm{Li}_{2} \mathrm{SiP}_{2}$.

The site 2 pathway in Figure 7 corresponds to an interstitial mechanism, instigated by the spontaneous creation of a lithium Frenkel pair. The Frenkel pair is created by a lithium ion moving from a lithium site 1 into an interstitial space in the lithium site 2 pathway, Figure $7 \mathrm{~b}$ ). The vacancy located on lithium site 1 is immediately filled by diffusion of another lithium atom from a lithium site 3 , thereby blocking the return of the original site 1 lithium, Figure $7 \mathrm{c}$ ). It is interesting to note that the AIMD pathway observed in Figure 7 b) does not show segmentation with higher density at the original Li sites, but is rather a continuous band of lithium density, suggesting the lithium ions in the pathway are not strongly bound to the original sites.

The same continuous band of lithium positions is always observed in $\mathrm{Al}_{\mathrm{Si}}^{\prime}$ doped LSP, Figure 8, suggesting the presence of $\mathrm{Al}_{\mathrm{Si}}^{\prime}$ defects do not act as a trap for diffusing lithium ions. This is unsurprising given the relatively small difference in charges between $\mathrm{Si}^{4+}$ and $\mathrm{Al}^{3+}$.

To probe this unusual pathway further, Nudged Elastic Band (NEB) calculations were performed within a pure LSP-160 simulation cell. ${ }^{40,41} \mathrm{Al}_{\mathrm{Si}}^{\prime}$ defects were not included in the NEB calculations due to the observed similarity with $\mathrm{Si}_{\mathrm{Si}}^{\mathrm{X}}$ (i.e. no clustering of defects) and to reduce the number of factors under consideration. From the AIMD data it is known that this pathway is usually activated by the presence of a lithium interstitial in the pathway, although stoichiometric and vacancy mechanisms were also probed. The NEB calculations show that the site 2 pathway is highly unlikely to be activated without defects present, as the activation barrier is on the order of $0.73 \mathrm{eV}$ for a stoichiometric mechanism. However, once a defect is present, whether vacancy or interstitial, the activation barrier drops significantly. The site 2 vacancy mechanism has an activation barrier on the order of $0.22 \mathrm{eV}$, indicating it is a feasible mechanism for lithium migration. The most striking result however is the activation barrier for the interstitial pathway. By inserting a single Li interstitial into the site 2 chain, the activation barrier drops even further to $0.05 \mathrm{eV}$. It is this extraordinarily low barrier to lithium migration which accounts for the smooth distribution of lithium seen in the site 2 pathway, Figure 7 . The migration pathway predicted for the interstitial site 2 pathway results in a very minimal disruption to the rest of the lattice, Figure 9, which somewhat accounts for the low energy barrier to migration. However, it must be noted that the site 2 pathway is 1-dimensional in the xy-plane and there may exist other low energy pathways for defects to move out of the pathway, significant increasing the activation energy of lithium migration along the pathway. Thus, the site 2 pathway may only be active under certain conditions, such as high Li content, and may only be active for relatively short periods of time and is therefore unlikely to dominate the 3-dimensional diffusion coefficients.

The vacancy component of the Frenkel pair formed in Figures 7 b) and c) travels via a much more complex pathway through the simulation cell than the interstitial component. Further NEB calculations have been performed into a number of lithium vacancy migration mechanisms, see SI. Importantly, the lithium vacancy mechanisms studied constitute a 3-dimensional network spanning the entire material. The majority of lithium vacancy migration mechanisms have activation energies of 0.2 $0.3 \mathrm{eV}$, in strong agreement with the AIMD calculations, suggesting the 3-dimensional vacancy mechanisms may be dominating the long-range transport of lithium ions, particularly in pure LSP.

It is therefore conceivable that the introduction of $\mathrm{Li}_{\mathrm{i}}^{\bullet}$ defects boosts the site 2 interstitial pathway at low temperatures and leads to higher ionic conductivity; but as the temperature is increased, spontaneous lithium Frenkel defects are able to form and the presence of additional $\mathrm{Li}_{\mathrm{i}}^{\bullet}$ defects leads to a suppression of the vacancy pathways and results in ionic conductivity values on-par with pure LSP.

\subsection{Electronic properties}

The $\mathrm{Al}$ doped system used contains a single $\mathrm{Al}$ defect and associated Li interstitial. No effect on the bandgap or the nature of the VBM or CBM was observed, see the SI. The bandgap is calculated to be approximately $2.4 \mathrm{eV}$ for both pure and doped systems.

It is also possible to calculate the voltage obtained by inserting or removing additional Li ions into the LSP structure. The voltage gained from intercalation of a single additional lithium into the LSP structure is given by Equation 8, where $V_{i n t}$ is the intercalation voltage, $\mathrm{E}(x)$ denotes the lattice energy of material $x, \mathrm{~F}$ is the Faraday constant and Li denotes metallic lithium.

$$
V_{\text {int }}=\frac{-\left[\mathrm{E}\left(\mathrm{Li}_{65} \mathrm{Si}_{32} \mathrm{P}_{64}\right)-\mathrm{E}\left(\mathrm{Li}_{64} \mathrm{Si}_{32} \mathrm{P}_{64}\right)-\mathrm{E}(\mathrm{Li})\right]}{\mathrm{F}}
$$

Likewise, Equation 9 gives the voltage obtained from deintercalating a single lithium from the LSP structure, $V_{\text {deint }}$. 

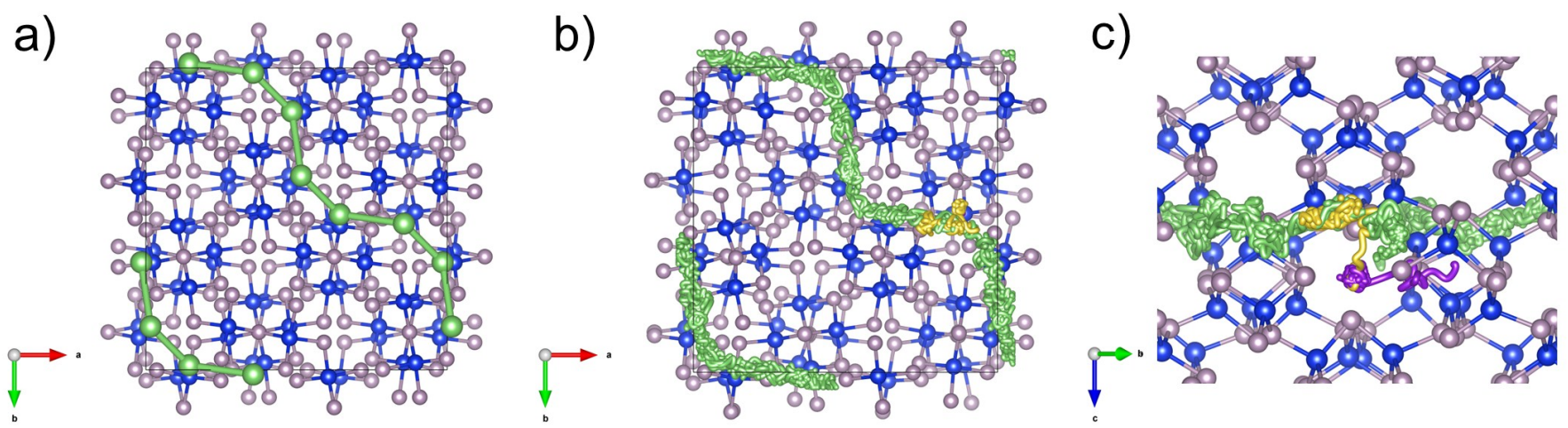

Fig. 7 The LSP-320 cell, displaying a) the lithium site 2 chain involved in the interstitial diffusion mechanism, b) the pathway extracted from the AIMD calculations and $\mathrm{c}$ ) the same pathway viewed from the side with instigating spontaneous lithium Frenkel defect.

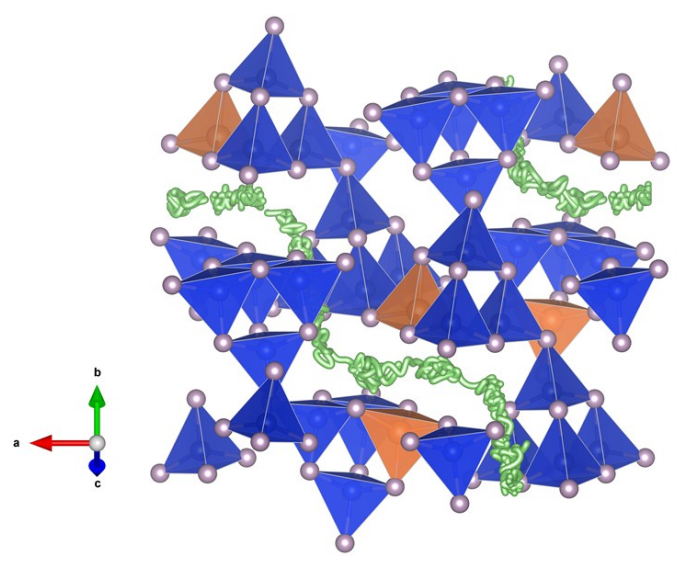

Fig. 8 A 2.5 ps segment of an AIMD simulation, showing the site 2 diffusion pathway in LSP-320 with $\mathrm{Al}_{\mathrm{Si}}^{\prime}$ defects. The simulation shows no clustering of lithium with the $\mathrm{Al}_{\mathrm{Si}}^{\prime}$ defects.

$$
V_{\text {deint }}=\frac{-\left[\mathrm{E}\left(\mathrm{Li}_{63} \mathrm{Si}_{32} \mathrm{P}_{64}\right)+\mathrm{E}(\mathrm{Li})-\mathrm{E}\left(\mathrm{Li}_{64} \mathrm{Si}_{32} \mathrm{P}_{64}\right)\right]}{\mathrm{F}}
$$

The voltage generated by adding a single $\mathrm{Li}$ to form $\mathrm{Li}_{65} \mathrm{Si}_{32} \mathrm{P}_{64}$ is approximately $-0.46 \mathrm{~V}$. The voltage generated by removing a single $\mathrm{Li}$ to form $\mathrm{Li}_{63} \mathrm{Si}_{32} \mathrm{P}_{64}$ is approximately $-2.72 \mathrm{~V}$. LSP is therefore stable against $\mathrm{Li}$ metal under standard conditions. It must be noted that these values are only for a single lithium intercalation/de-intercalation process and the behaviour of the redox potential at greater/lesser lithium content is unknown.

\section{Conclusions}

In conclusion, trivalent doping of $\mathrm{Li}_{2} \mathrm{SiP}_{2}$ is most likely to occur on the $\mathrm{Si}^{4+}$ site rather than the $\mathrm{Li}^{+}$site. Aluminium doping on the silicon site with a charge compensating lithium interstitial is an extremely low energy doping mechanism with an energy barrier to incorporation of $\sim 0.05 \mathrm{eV}$. Via this mechanism, the charge compensating $\mathrm{Li}_{\mathrm{i}}^{\bullet}$ species instigates increased lithium diffusion. Furthermore, doping of up to $\sim 9 \% \mathrm{Al}_{\mathrm{Si}}^{\prime}$ is possible with very little change in energy per defect pair $(+0.01 \mathrm{eV})$.

The AIMD simulations reveal a high throughout pathway for $\mathrm{Li}^{+}$diffusion involving only lithium on site 2 . This highly curved

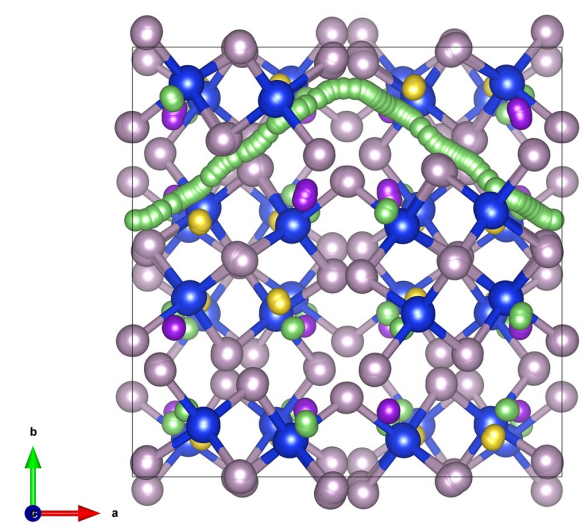

Fig. 9 The interstitial site 2 pathway as calculated by NEB in the LSP-160 simulation cell with a migration barrier of $0.05 \mathrm{eV}$. Note the minimal disruption to the rest of the lattice during the migration.

pathway is however only observed to activate upon migration of a lithium interstitial into the pathway. NEB calculations support the activation of the high-throughput pathway by interstitial $\mathrm{Li}$, showing an exceptionally low energy barrier to migration of $0.05 \mathrm{eV}$ when interstitial $\mathrm{Li}$ is included. The migration barriers were significantly higher in the site 2 pathway when the instigating mechanism is instead stoichiometric hops or vacancy hops.

Activation of the site 2 pathway, potentially by intrinsic as well as extrinsic defects, may be the origin of the high ionic conductivity observed experimentally. Future work will be concerned with exploring alternative doping schemes with an eye to generating lithium vacancies (with $\mathrm{Mg}^{2+}$ ). With diffusion data from lithium vacancies it then becomes possible to definitively ascertain any anisotropy in diffusion arising from either interstitial or vacancy lithium defects.

\section{Acknowledgements}

The authors acknowledge the support of EPSRC SUPERGEN grant, EP/N001982/1. The authors are grateful for the use of the 'Hydra' High Performance System at Loughborough University. via our membership of the UK's HEC Materials Chemistry Consortium, which is funded by EPSRC (EP/L000202, EP/R029431), 
this work used the ARCHER UK National Supercomputing Service (http://www.archer.ac.uk). We strongly acknowledge the use of Athena as part of the HPC Midlands + consortium, funded by the EPSRC on grant EP/P020232/1.

\section{References}

1 J. G. Kim, B. Son, S. Mukherjee, N. Schuppert, A. Bates, O. Kwon, M. J. Choi, H. Y. Chung and S. Park, Journal of Power Sources, 2015, 282, 299-322.

2 N. Kamaya, K. Homma, Y. Yamakawa, M. Hirayama, R. Kanno, M. Yonemura, T. Kamiyama, Y. Kato, S. Hama, K. Kawamoto et al., Nature materials, 2011, 10, 682-686.

3 Y. Mo, S. P. Ong and G. Ceder, Chemistry of Materials, 2011, 24, 15-17.

4 Y. S. Jung, D. Y. Oh, Y. J. Nam and K. H. Park, Israel Journal of Chemistry, 2015, 55, 472-485.

5 A. Manthiram, X. Yu and S. Wang, Nature Reviews Materials, 2017, 2, 16103.

6 C. Sun, J. Liu, Y. Gong, D. P. Wilkinson and J. Zhang, Nano Energy, 2017, 33, 363-386.

7 F. Zheng, M. Kotobuki, S. Song, M. O. Lai and L. Lu, Journal of Power Sources, 2018, 389, 198-213.

8 A. Haffner, T. Bräuniger and D. Johrendt, Angewandte Chemie International Edition, 2016, 55, 13585-13588.

9 L. Toffoletti, H. Kirchhain, J. Landesfeind, W. Klein, L. van Wüllen, H. A. Gasteiger and T. F. Fässler, Chemistry-A European Journal, 2016, 22, 17635-17645.

10 H. Eickhoff, L. Toffoletti, W. Klein, G. Raudaschl-Sieber and T. F. Fässler, Inorganic Chemistry, 2017.

11 B. Eisenmann and M. Somer, Zeitschrift für Naturforschung. Teil b, Anorganische Chemie, organische Chemie, 1985, 40, 886-890.

12 A. Haffner, A.-K. Hatz, I. Moudrakovski, B. V. Lotsch and D. Johrendt, Angewandte Chemie, 2018, 130, 6263-6268.

13 B. Eisenmann and M. Somer, Zeitschrift für Naturforschung. Teil b, Anorganische Chemie, organische Chemie, 1984, 39, 736-738.

14 K. Feng, L. Kang, W. Yin, W. Hao, Z. Lin, J. Yao and Y. Wu, Journal of Solid State Chemistry, 2013, 205, 129-133.

15 A. Springthorpe and J. Harrison, Nature, 1969, 222, 977-977.

16 S. Pagano, M. Zeuner, S. Hug and W. Schnick, European Journal of Inorganic Chemistry, 2009, 2009, 1579-1584.

17 G. Kresse and J. Hafner, Physical Review B, 1994, 49, 14251.

18 P. E. Blöchl, Physical review B, 1994, 50, 17953.

19 J. P. Perdew, A. Ruzsinszky, G. I. Csonka, O. A. Vydrov, G. E. Scuseria, L. A. Constantin, X. Zhou and K. Burke, Physical
Review Letters, 2008, 100, 136406.

20 A. V. Krukau, O. A. Vydrov, A. F. Izmaylov and G. E. Scuseria, The Journal of chemical physics, 2006, 125, 224106.

21 M. P. Allen and D. J. Tildesley, Computer simulation of liquids, Oxford university press, 2017.

22 M. Parrinello and A. Rahman, Physical Review Letters, 1980, 45, 1196.

23 M. Parrinello and A. Rahman, Journal of Applied physics, 1981, 52, 7182-7190.

24 S. Nosé, The Journal of chemical physics, 1984, 81, 511-519.

25 W. G. Hoover, Physical review A, 1985, 31, 1695.

26 G. Eulenberger, D. P. Shoemaker and J. Keil, The Journal of Physical Chemistry, 1967, 71, 1812-1819.

27 T. Xue, H. Liu, Y. Zhang, H. Wu, P. Wu and M. He, Microporous and Mesoporous Materials, 2017, 242, 190-199.

28 R. M. Barrer and E. F. Freund, Journal of the Chemical Society, Dalton Transactions, 1974, 1049-1053.

29 M. V. Shamzhy, C. Ochoa-Hernández, V. I. Kasneryk, M. V. Opanasenko and M. Mazur, Catalysis Today, 2016, 277, 3747.

30 R. Fricke, H. Kosslick, G. Lischke and M. Richter, Chemical reviews, 2000, 100, 2303-2406.

31 H. Xia, Q. Xia and A. L. Ruoff, Journal of applied physics, 1993, 74, 1660-1662.

32 D. Richman, Journal of The Electrochemical Society, 1968, 115, 945-947.

33 M. K. Rabadanov, A. Loshmanov and Y. V. Shaldin, Crystallography Reports, 1997, 42, 592-602.

34 J. Čejka and R. E. Morris, Zeolites in catalysis: properties and applications, Royal Society of Chemistry, 2017, vol. 28.

35 R. D. Shannon, Acta crystallographica section A: crystal physics, diffraction, theoretical and general crystallography, 1976, 32, 751-767.

36 G. Murch, Solid State Ionics, 1982, 7, 177-198.

37 P. M. Panchmatia, A. Orera, G. J. Rees, M. E. Smith, J. V. Hanna, P. R. Slater and M. S. Islam, Angewandte Chemie, 2011, 123, 9500-9505.

38 B. J. Morgan, Royal Society open science, 2017, 4, 170824.

39 J. Isard, Journal of non-crystalline solids, 1999, 246, 16-26.

40 G. Mills, H. Jónsson and G. K. Schenter, Surface Science, 1995, 324, 305-337.

41 H. Jónsson, G. Mills and K. W. Jacobsen, Classical and quantum dynamics in condensed phase simulations, World Scientific, 1998, pp. 385-404. 
The ionic conductivity and diffusion pathways of the supertetrahedral lithium phosphidosilicate $\mathrm{Li}_{2} \mathrm{SiP}_{2}$ are studied alongside the impact of trivalent doping.

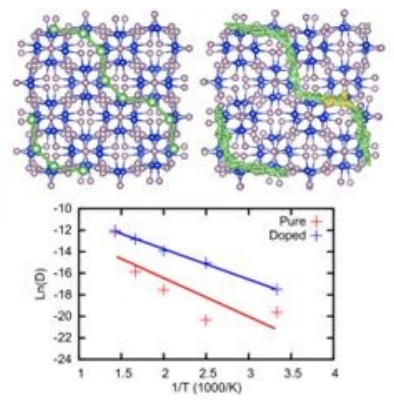

